\title{
Impeccable Way of Administering Anesthesia using Brain Wave Sensor
}

\author{
S. Jagadeesh Babu, D.S.Bhargava, T.V.Padmavathy, T. Blesslin Sheeba
}

\begin{abstract}
In the hospitals, during major operation the patient should be anesthetized for pain free operation. Single dose anesthesia administration for prolonged operations may lead to complications. In case of insufficient anesthesia dose, the patient may feel pain and discomfort during operation. Therefore, there is need for an administration of exact dosage to the patient, at the particular time interval. An automatic anesthesia administering machine is proposed to overcome the crucial issues. The anesthesia should be given to patient in the correct dose at regular interval based on patient conscious state. The brainwave sensor is placed on the frontal side on the scalp. Brains action will be monitored constantly. When the patient gets deviated from the sleep state the correct level of anesthesia is injected by using servo motor by analyzing the brain activity. The microcontroller and brainwave sensor are interlinked using bluetooth module where the data are processed and sent to raspberry pi which gives commands to servo motor to pump the required amount of anesthesia to the patient. In addition to this heart rate and temperature of the individual are recorded.
\end{abstract}

Keywords: Arduino, Bluetooth module, Electro Encephalogram, Servomotor.

\section{INTRODUCTION}

In recent times medical electronics have gained at most attention because it encompasses a variety of health care practices evolved to maintain and restore health by prevention and treatment of illness. These advancements lead to cure of various fatal diseases by application of engineering. This idea signifies the general domain of remote patient monitoring and focused on the anesthesiology care domain. Anesthesia is a kind of sedative administered into the patient for doing pain free operation. Administering anesthesia requires more complex technical equipment and constant monitoring by a skilled anesthetist. It keeps the patient in unconscious state. It allows stable performance of medical and surgical procedures.

The patient's conscious is monitored using sensor during surgery. This will facilitate anesthetists to regulate drug delivery and ensure patients stay painless and unconscious

Revised Manuscript Received on November 27, 2019.

* Correspondence Author

S.Jagadeesh Babu, Assistant Professor, Department of ECE in R.M.K. Engineering College, Mail: sjb.ece@rmkec.ac.in

D.S. Bhargava, Assistant professor in ECE in R.M.K Engineering College, Mail: dsb.ece@ rmkec.ac.in

T. V. Padmavathy, Professor, Department of ECE in R.M.K. Engineering College, Mail: tvp.ece@rmkec.ac.in

Dr.T.Blesslin Sheeba, Professor, Department of ECE in R.M.K Engineering College, Mail: tbs.ece@rmkec.ac.in till they are supposed to. This is important as inadequate anesthetic may lead to discomfort during surgery. On the contrary, too much anesthetic can result in a patient taking longer time to recover from surgery.

The sensors on the scalp sense the signals of brain activity. The degree of alertness of the individual is determined by how the aspects of brain areas communicate within itself and is measured with the help of EEG. Induction, Maintenance, and Emergence are the different stages of anesthesia in EEG. Alpha activity (10 Hertz) is measured in EEG before induction of anesthesia [5]. Anesthesia administration may lead to physiological changes in patients such as blabbering of speech, euphoria or dysphoria and confusion in time perception which are the results of increased in beta activity (13-25 Hertz) [2].

This phase ends with LOC (Level of consciousness), in which the patient shows unconsciousness, depression of brain-stem reflexes, no response to stimuli, apnoea, and the need for cardio respiratory and thermoregulatory support. Different Electroencephalogram (EEG) pattern is observed during anesthesia injections, based on the DoA (Depth of Physiological State). We process the EEG patterns and accordingly we inject anesthesia to the patient during a complex surgery[3].

\section{RELATED WORKS}

The slow waves observed in EEG are used for monitoring the intensity of anaesthesia for the patients. The rapid changes in the EEG slow wave was analysed with the help of Hilbert-Huang Transform. It is a data-driven adaptive method which is used to take out stable signal components signifying slow wave activity. Based on the data gathered from signal transform, possible specific structure between different components dependent on the strength of anaesthesia was not addressed in [7].

The idea of a Smart Medical System applied to the general domain of remote patient monitoring and focused on the anaesthesiology care domain. Based on the state-of-the art of the research on patient monitoring techniques, a cutting-edge solution to perform DoA [4] monitoring activities during the intra-operative period in a smart, remote, autonomous and wireless way, by using EEG sensors and software technology. The importance of regulating the consumption of anesthetic agents, by reducing excessive dosage administration or increasing insufficient dosage, aiming to shorten the anesthetic recovery period, as well as the adverse effects of 
anaesthesia. One in all the objectives of contemporary physiological state is to confirm adequate depth of physiological state to stop awareness while not unwittingly overloading the patients with potent medicine. The achievements of contemporary physiological state is the ability to observe depth of physiological state. Measure depth of physiological state represents the foremost debatable and subjective facet of contemporary physiological state, with the introduction of the idea of balanced physiological state exploitation multiple medicine and muscle relaxants. It's not likely that any single methodology is going to be found to live the depth of physiological state dependably for every patients and every one anaesthetic agent. All the strategies for decisive depth of physiological state thus far have some potential exclusion criteria. Therefore, exploitation of one methodology at a time could offer a lot of accuracy [1].

The bispectral index (BIS) monitor could be a quantitative encephalogram device that's wide work to measure the hypnotic element of physiological state, particularly once fascicle interference medicine area unit used. it's been shown that the BIS [6] is sensitive to changes is myogram activity in anesthetized patients. one study exploitation as past versions of the BIS showed that ablated myogram action caused the BIS to reduce even i very awake subjects, to level that steered deep sedation and physiological state. To change a DoA to observe the proper estimation across a variety of patients, a unique feature extraction together with machine learning processor is used[1]. An easy call tree is employed to perform a multiclass DoA classification. The projected patient- specific DoA classification processor achieves a classification accuracy of seventy-nine.

Typical systems use wired information transfer for communication between sensing element and also the main system. Thus, the prevailing systems have strained distance of information transfer. The projected system resolves this drawback to get convenience and movability. With clinical trials, top quality signal wireless transmission is verified [10].

Accurate and noninvasive observance of the depth of physiological state (DoA) is extremely fascinating. Since the anesthetic medicine act principally on the central nervous system, the analysis of brain activity exploitation graphical record (EEG) is extremely helpful. This paper proposes a unique machine-controlled methodology for assessing the DoA exploitation encephalogram. It tends to feed these extracted options to a brand new neurofuzzy classification formula, accommodative neurofuzzy logical thinking system with linguistic hedges. The bestowed methodology classifies encephalogram information into awake, light, general, and deep states throughout physiological state with sevoflurane in seventeen patients. Its accuracy is ninety two compared to a regular observance system [9].

\section{PROPOSED SYSTEM METHODOLOGY}

Based on the brainwave we are able to analyze the patient's consciousness. The heart rate, pressure level of the patient is monitored and consequently the drug is used. If the EEG signal is rising up then anaesthesia injection is required more than once. In rural areas continuous monitoring of patients could be a tough task because the skilled anesthetist are less when compared to those in cities. Therefore, the remote watching is feasible only via knowledge sharing by analyzing the brainwaves

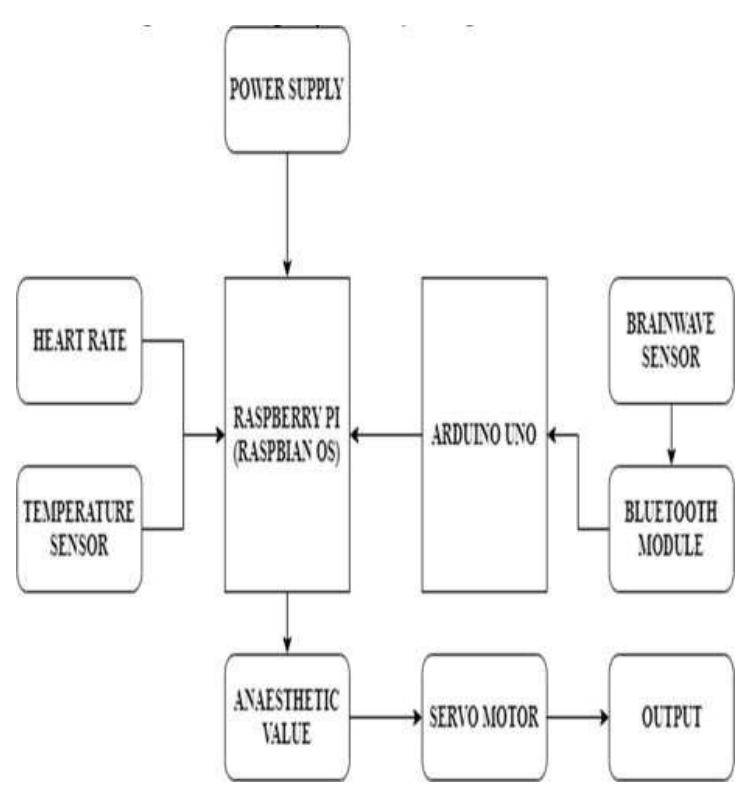

Fig. 1 Block Diagram for Automated Anesthesia Administration

The brainwave device securely measures and produces the EEG power spectrums (alpha waves, beta waves, etc.), Brainwave visualizer shows the amount of attention through eyelid blinking. The appliance consists of a telephone receiver, associate degree ear-clip, and a device arm. The headband's reference and earthing conductors are attached on the clip which is placed on the earlobe and also the EEG electrode is on the device arm, placed on the forehead on top of the attention (FP1 position). It use one aortic aneurysm battery with a eight hour battery life

\section{HARDWARE DETAILS}

Computer hardware is that the assortment of physical parts that represent component. Component is that the physical elements or parts of a laptop, like monitor, keyboard, laptop storage, graphical card, sound card, motherboard, etc. Against this, software package is directions which will be hold on and travel by hardware. 


\section{A. Raspberry pi}

The Raspberry Pi may be a low value, visiting- card sized processor that can be plugged into a laptop or TV and uses a regular keyboard and mouse. It is a highly capable testbed using python and widely used in embedded domain. It's a miniature processor capable of doing many functions such as browsing the net and enjoying high-definition video, to creating spreadsheets, data processing and enjoying games.

\section{B. Arduino UNO}

Arduino Uno is a microcontroller having fourteen digital in/out pins, cardinal analog inputs, a USB affiliation, an influence jack, ICSP header and a push. It is easy to interface with micro-controller. In italian "Uno" refers to one and it was taken as the base for naming of Arduino code as (IDE) one.0. Higher versions of UNO are periodically replacing the older versions for enhanced support.

\section{Brainwave Sensor}

Thoughts, Emotional feelings and Behavioural responses are the communication between various neurons of the Human Brain. Brainwaves sensor placed on the head measure the synchronal electrical pulses associated with neurons. The results is separated into bandwidths to indicate the patient's level of awareness; from slow, loud and useful - to quick, subtle, and complicated. Brainwave sensor is powered by a separate source (battery) since it is connected wireless to Arduino via Bluetooth. The Arduino processes the output waves of brainwave sensor and forwards the information to Raspberry pi.

\section{Bluetooth}

It is a class-2 bluetooth module with interface Profile, which may put together as either Master or slave. It's an alternative for wired serial connections and used as an interface replacement to ascertain affiliation between HC-05 Specification.

\section{E. Heart Beat Sensor}

This device produces binary output of heart beat once a finger is positioned on that. The beat junction rectifier flashes in unison for every heartbeat. This live digital output connected to microcontroller displays Beats Per Minute (BPM) rate. It works on the concept of sunshine modulation by blood movement in finger for every pulse.

\section{F. Temperature Sensor}

DHT11 Temperature sensing element options a temperature sensing element complicated with a mark digital signal output. The elite digital-signal technique and warmth sensing technology ensures high dependability and glorious long-run stability. This sensing element includes a resistive-type humidness activity element associated with an NTC temperature activity element, and connects to a highly performing 8-bit microcontroller, which is cost effective and less susceptible to interferences.

\section{EXPERIMENTAL SETUP}

The flow diagram in Fig. 2 indicates the sequences of processes happening in the prototype developed. The brainwave sensor is paced on the patients scalp and the readings recorded are transferred to the arduino processor via Bluetooth. The outputs from the temperature sensor and heart beat sensor are also transmitted to the processor. The servo motor setup mimics the induction of anesthesia. The threshold for the servomotor to start and stop operation is setup as per the alpha wave values got from brainwave sensor.

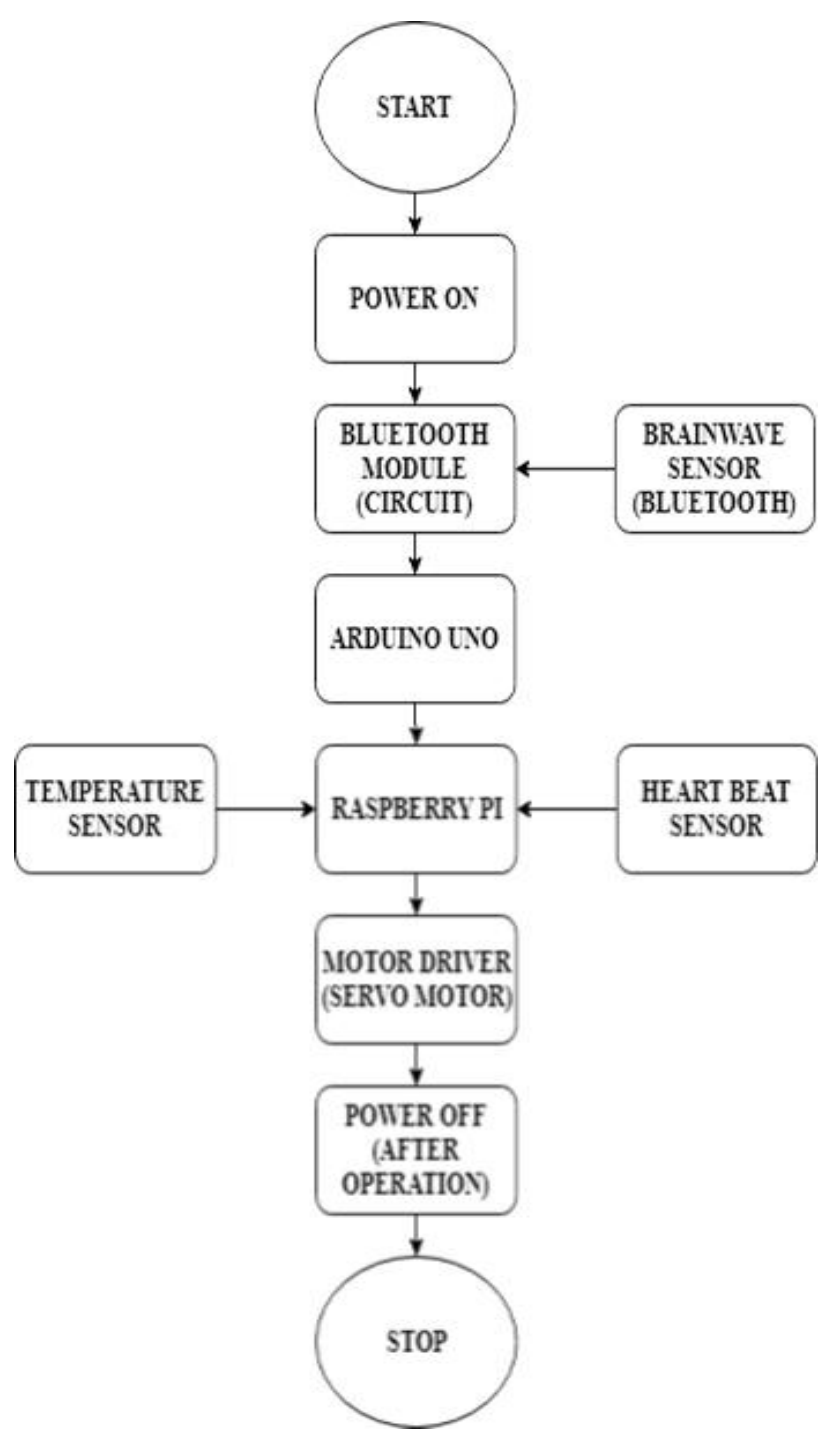

Fig. 2 Methodology for Automated Anesthesia Administration

The prototype developed is shown in Fig.3. The brainwave sensor is placed in a belt which can be worn around the scalp. The remaining hardware is placed in a separate board which hosts the processor and the motor that is mimicking the anesthesia administration procedure. 


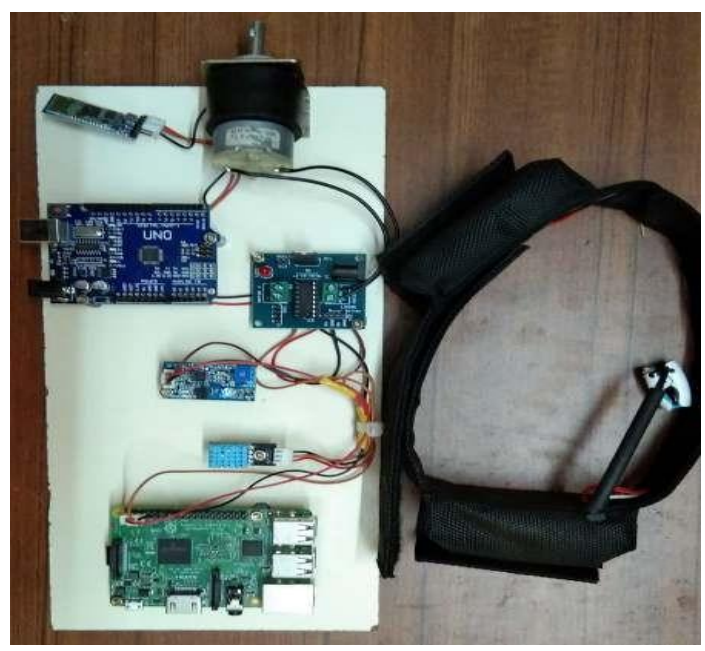

Fig. 3 Experimental Setup for Automated Anesthesia Administration

\section{CONCLUSION AND FUTURE SCOPE}

This paper helps us to support and supplement the anesthetist nature of work to reduce frequent monitoring of patients who are undergoing surgery. It can be used in treasury level hospitals, wherein well trained professionally enriched anesthetists are not available. Further it was hypothesized that human attention span is less when compared to modern equipment which are programmed to address the fatigueness of humans. Merits and limitations of EEG signal detection along with decomposition process, practicality and classification were discussed. Thus far to our understanding no monitoring system are capable of DoA and are attuned to patients and all anesthetist agents but the existing monitors adequately provide productive signal for doctors regarding the patient's conditions in the surgical theatre.

It is anticipated that in next decade anesthesia administration would be computerized. Anesthesia machines will be verbally interactive in nature. Notifications are also performed automatically by software agents and can be sent through SMS, email or Bluetooth messages. As a result of the monitoring phase of EEG, the effective detection of anomalies in EEG data and consequent notifications to the professionals involved.

\section{REFERENCES}

1. Margalit, E., Weiland, J., Clatter buck, R., Fujii, G., Maia, M., Tameesh, M., Torres, G., D’Anna, S., Desai, S., Piyathaisere, D., Olivi, A., de Juan, E.J., Humayun, M.: Visual and electrical evoked response recorded from subdural electrodes implanted above the visual cortex in normal dogs under two methods of anesthesia. J. Neurosci. Methods 123(2), 129-137 (2003)

2. Marple, S.L.: Digital Spectral Analysis: With Applications. Prentice-Hall, Englewood Cliffs (1987)

3. McFarland, D.J., Neat, G.W., Wolpaw, J.R.: An EEG-based method for graded cursor control. Psychobiol. 21, 77-81 (1993)

4. McFarland, D.J., Lefkowicz, T.Wolpaw, J.R.: Design and operation of an EEG-based brain- computer interface (BCI) with digital signal processing technology. Behav. Res. Methods Instrum. Comput. 29, 337-345 (1997)

5. McFarland, D.J., McCane, L.M., David, S.V., Wolpaw, J.R.: Spatial filter selection for EEG based communication. Electroencephalogr. Clin. Neurophysiology. 103(3), 386-394 (1997)

6. McFarland, D.J., Miner, L.A., Vaughan, T.M., Wolpaw, J.R.: Mu and beta rhythm topographies during motor imagery and actual movements. Brain Topogr. 12(3), 177-186 (2000)

7. McFarland, D., Anderson, C.W., Müller, K.R., Schlögl, A., Krusienski, D.J.: BCI meeting 2005 - workshop on BCI signal processing: feature extraction and translation. IEEE Trans. Neural Syst. Rehabil. Eng. 14(2), 135-138 (2006)

8. McFarland, D.J., Krusienski, D.J.,Sarnacki, W.A., Wolpaw, J.R.: Emulation of computer mouse control with a noninvasive brain-computer interface. J. Neural Eng. 5(2), 101-110 (2008). doi:10.1088/1741-2560/5/2/001.

9. Mellinger, J., Schalk, G., Braun, C., Preissl, H., Rosenstiel, W., Birbaumer, N., Kübler, A.: An MEG-based brain-computer interface (BCI). NeuroImage 36(3), 581-593 doi:10.1016/j.neuroimage.2007.03.019

10. Millán, J. del R., Renkens, F., Mouriño, J., Gerstner, W.: Noninvasive brain- actuated control of a mobile robot by human EEG. IEEE Trans. Biomed. Eng. 51(6), 1026-1033 (2004)

\section{AUTHORS PROFILE}

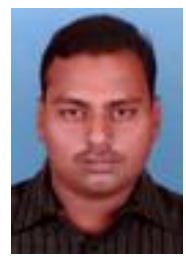

S.Jagadeesh Babu, Assistant Professor, Department of ECE in R.M.K. Engineering College, has 10 years of teaching and research experience in the in the fields of VLSI Design, Wireless sensor networks and Wireless Communication. He has graduated from Adhiparasakthi Engineering College, in Electronics and Communication Engineering. He has obtained his Master degree in VLSI Design from Easwari Engineering, Chennai. His current area of research includes Low power VLSI and proficient in backend tool CADENCE. He is a member of various professional bodies such as Life member of Institution of Electronics and Telecommunication Engineers (IETE), International Association of Engineers (IAENG), and Life member of Indian Society for Technical Education ISTE.

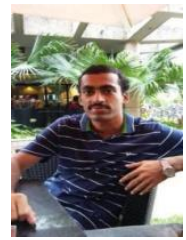

D.S. Bhargava Assistant professor in Electronics and Communication Engineering department of R.M.K Engineering College, has 5 years of teaching experience. He received his Bachelor's degree from J.N.N Institute of Engineering in Electronics and Communication Engineering in the year 2012 and Master's degree in VLSI Design from R.M.K Engineering College in the year 2014. His area of interests includes Cognitive radio networks and VLSI Design technology. He has published 7 research papers in International and National Journals and conferences in the area of VLSI Design, Networking and Cognitive radio networks. He is a Life member of ISTE.

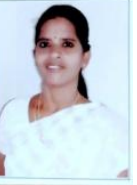

T. V. Padmavathy Professor, Department of ECE in R.M.K. Engineering College, has 24 years of teaching and research experience in the in the fields of Wireless sensor networks, Under Water Acoustic Sensor Networks and Antenna Design. She has graduated from Institution of Engineers (India), in Electronics and Communication Engineering. She has obtained her Master degree in Control and Instrumentation from College of Engineering, Guindy, Anna University, Chennai and Ph.D. degree from Anna University, Chennai. She has published more than 50 research papers in International and National Journals and conferences in the area of Mobile Ad hoc Networks, Wireless sensor networks, Under Water Acoustic Sensor Networks and Antenna design and she has four Patents in Wireless Sensor Networks. Her current area of research includes security and architecture issues of Mobile ad hoc networks, Wireless sensor networks and Millimeter Wave Antenna design for Wireless Communications. She is a technical paper reviewer for African Journal of Engineering Research and Journal of Engineering and Technology Management. She is recognized as Fellowship member by The Institution of Engineers (India) also she is a member of various professional bodies such as Institute of Electrical and Electronics Engineers (IEEE), Life member of Institution of Electronics and Telecommunication Engineers (IETE), International Association of Engineers (IAENG), ACM, ISSE and Life member of Indian Society for Technical Education ISTE.

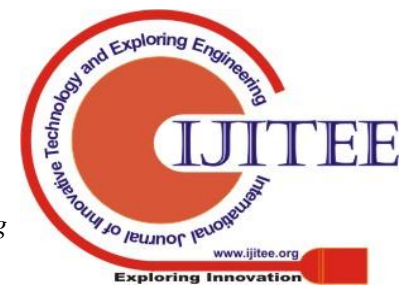




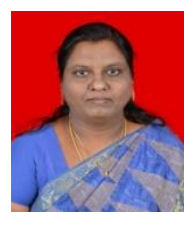

Dr.T.Blesslin Sheeba received her Ph.D in Crytptographic Algorithms for Small Embedded Applications from Sathyabama University, Chennai. She is working as Professor in the department of ECE, RMK Engineering College, Chennai. She has 26 years of Teaching and research experience. She has published more than 25 research papers in International and National Journals. Her current area of research includes security and architecture issues in networks and Antenna design. She is recognized as Fellowship member by The Institution of Engineers (India) also she is a member of various professional bodies such as Life member of Indian Society for Technical Education ISTE, IACST, ACM and ISSE. 